\title{
FUNDAMENTACIÓN DEL DEPORTE SOCIAL COMUNITARIO A PARTIR DE LAS CATEGORÍAS BIOÉTICAS: UNA OPCIÓN HACIA EL MEJORAMIENTO DE LA CALIDAD DE VIDA QUE TRASCIENDE EL DEPORTE MODERNO ${ }^{1}$
}

\author{
FOUNDATIONS OF COMIUNITARIAN SPORT FROM BIOETHICS CATEGORIES: AN OPTION \\ TOWARDS THE IMPROVEMENT OF THE QUALITY OF LIFE THAT EXTENDS MODERN SPORT
}

\section{Resumen}

\begin{abstract}
La tesis central de esta reflexión teórica consiste en que el deporte social comunitario es un proceso diferente y de más trascendencia que el deporte moderno, en tanto que se fundamenta y operativiza en la construcción colectiva y constante de tejido comunitario y usa como herramienta el deporte y las transformaciones que hace de él. Este proceso implica dinámicas de reflexión y acción a partir de un análisis desde las categorías bioéticas en torno a las necesidades de las comunidades en donde se pone en juego la calidad de vida. Las categorías desde donde se hace este análisis son la interdisciplinariedad y la transdisciplinariedad, el pluralismo, la reflexión, la deliberación, el diálogo, el respeto por la existencia de diferentes grados de libertad, la colectividad y la comprensión del contexto. Contrario a lo que ocurre en el deporte moderno, en el deporte social comunitario la vida se ubica como eje central de reflexión y de acción en busca de que los seres humanos desarrollen relaciones comprensivas consigo mismos, con los otros y con el contexto a partir de la deliberación, el diálogo y el desarrollo de la conciencia.
\end{abstract}

Palabras clave: bioética, deporte social comunitario, calidad de vida, pluralismo, conciencia.

Abstract

The thesis maintained and argued within the following pages is that Communitarian Sport is a different and more important process than Modern Sport whereas it's founded and operationalized on the collective and constant construction of communitarian network using sport and the transformations it causes as a tool. This process implies dynamics of reflection and action through an analysis that uses bioethics categories to approach communities' necessities where quality of life is compromised. The categories used for this analysis are interdisciplinarity and transdisciplinarity, pluralism, reflection, deliberation, dialogue, respect towards the existence of different degrees of freedom, and understanding of the context. Contrary to what happens in Modern sport, in Communitarian Sport life is deemeda central axis of reflection and action that pursues that human beings develop relations of understanding with themselves, with others and with the context, founded on deliberation, dialogue and development of the conscience.

Keywords: bioethics, communitarian sport, quality of life, pluralism, conscience.

1 Reflexión teórica realizada como parte de la investigación titulada "Deporte social comunitario: una opción para el mejoramiento de la calidad de vida". Esta investigación se desarrolla en el marco del Grupo de Investigación y Estudios Socioculturales del Deporte, la Educación Física y la Recreación (GRIESDE), de la Universidad Libre de Colombia y como opción de grado de la Maestría en Bioética de la Universidad El Bosque.

2 Magister en Bioética. Especialista en Comunicación - Educación. Licenciado en Educación Física. Profesor-Investigador, Universidad libre de Colombia. Correo electrónico: ridubaco@gmail.com. Página web: www.wix.com/ridubaco/index 


\section{Introducción}

En esta reflexión teórica se presentan de manera sintética algunos criterios para poder desarrollar una fundamentación del deporte social comunitario a partir de las categorías bioéticas, lo que permite que ese deporte pueda ser una opción que trasciende el deporte moderno y que se oriente hacia el mejoramiento de la calidad de vida. Las categorías bioéticas a las que aquí se hace referencia y que se explicarán sintéticamente más adelante son: transdisciplinariedad, pluralismo, reflexión, deliberación, diálogo, respeto por la existencia de diferentes grados de libertad, colectividad y comprensión del contexto. Así mismo, tres conceptos construidos desde la Bioética, gracias a las categorías mencionadas, permitirán realizar la fundamentación aquí propuesta; el concepto de extraños morales y la idea de comunidad de orden superior, expuestos por Tristram Engelhardt, y el principio de igual consideración de intereses, presentado por Peter Singer.

Esta reflexión teórica se organiza en dos partes; en la primera se realiza una rápida revisión de las transformaciones que han existido en la relación deporte-sistemas de valores, hasta llegar a lo que se conoce como deporte para todos. Posteriormente, se exponen las características que puede tener el deporte social comunitario si se fundamenta desde las categorías bioéticas y desde tres conceptos emanados de la Bioética.

\section{Antecedentes y quiebres dentro de la relación poder - ética - vida: del deporte moderno al deporte social comunitario}

Con el ánimo de establecer la problemática que sostiene este trabajo, es conveniente realizar una rápida revisión del impacto que el deporte ha tenido sobre la vida. El deporte moderno ha pasado por varios quiebres; cada uno de ellos evidencia un sistema axiológico con un determinado uso de fuerzas sobre la vida. Un primer quiebre se ubica en el tránsito de un poder disciplinario sobre el individuo a un poder regulador sobre la especie (Foucault, 1994) en donde se privilegia con mayor ímpetu la ganancia, la productividad y el éxito. Éste es un proceso característico de lo que se ha denominado deporte moderno y que tiene su origen durante los siglos XVIII y XIX (Mandell, 1986; véase también Velázquez Buendía, 2001).

Un segundo quiebre tiene que ver con la polarización política ocasionada por el auge de sistemas autoritarios
(Gonzalez, 2002). En estos escenarios, el deporte fue utilizado de diferentes maneras; por ejemplo, en Italia, hasta finales de la década de 1920, el deporte sirvió exclusivamente como un medio de formación física de su población. Después de 1929, en ese país y durante todo el proceso de ascenso de los totalitarismos en Alemania y en España, el deporte fue usado como un medio propagandístico en dos sentidos; primero, para promulgar su ideología dentro de la población, enfatizando los valores ya mencionados. En segundo término, el deporte fue usado para divulgar la fortaleza y progreso de la nación en el contexto mundial (Gonzalez, 2002; Teja, 2002). Años después se aprobó la Declaración Universal de los Derechos Humanos, con la que se buscó transformar los sistemas de valores y los conceptos de fuerza y convivencia, a la vez que privilegiar la valoración de la libertad, la justicia y la paz en el mundo. En este contexto, el deporte adquiere nuevos enfoques axiológicos como se verá a continuación.

Un tercer quiebre tiene que ver con el auge del deporte al que se pudiera vincular la mayoría de la población. Por esta vía y en algunas latitudes se buscaba el desarrollo de un espíritu combativo y aguerrido, entre otros valores. Cabe referenciar aquí el planteamiento realizado por Fidel Castro en la inauguración de la ciudad escolar 26 de julio (antiguo Cuartel Moncada), el 28 de enero de 1960. Refiriéndose a las nuevas perspectivas que ofrecía la revolución para los niños, expresó:

Ustedes tienen la oportunidad de hacer deporte, ¿ustedes saben cómo nosotros aprendimos a hacer la guerra? No vayan a creer que nosotros aprendimos a hacer la guerra en la Sierra Maestra; nosotros aprendimos a hacer la guerra cuando éramos muchachos igual que ustedes. ¿Saben cómo? ¿Quieren que les diga cómo? Bueno, nosotros aprendimos a hacer la guerra, jugando pelota, jugando básquet, jugando fútbol, haciendo todos los deportes, nadando en el mar, nadando en los ríos y subiendo montañas (Ruiz Aguilera, 1991: 95).

Evidentemente, dentro de este tercer quiebre se fue consolidando, de una parte, un deporte - espectáculo cuya función era cautivar a la mayor cantidad de público en torno a un proceso deportivo. Para lograrlo, se empezó a poner un enorme énfasis en su difusión y propaganda y se articuló de una manera avasalladora el deporte con la empresa, la publicidad y la política. De otro lado, se consolidó paulatinamente otro deporte, no muy cercano a la propaganda y a la comercialización masiva. En 1970, este deporte fue denominado el segundo camino del deporte, en la República Federal Alemana. 
Surgió entonces la idea de deporte para todos; se buscaba que la mayor cantidad de personas practiquen deporte buscando el desarrollo y la promoción de la salud y de valores morales. El cuarto quiebre se fundamentó en las teorías críticas; sus planteamientos argumentan que el deporte promueve de manera soterrada unos valores capitalistas que atentan contra la humanidad y contra la vida (Brohm, 1982).

Con base en el tercer quiebre, se hizo muy frecuente el uso del deporte como un medio para ayudar en el desarrollo social y comunitario. En este sentido, se adjetivizó el deporte, llamándolo formativo, recreativo, infantil, etc. Vale la pena aclarar que, por ejemplo, desde la ley 181, en Colombia el deporte se desarrolla de ocho formas (Constitución política de Colombia, 1995), pero solamente una de ellas tiene como objetivo el desarrollo de la calidad de vida: el deporte social comunitario.

Sin embargo, la reflexión que se hace en torno a este deporte es muy precaria y frecuentemente se ubica en el funcionalismo (Gonzalez, 2002); es decir, en una interpretación del deporte que lo usa exclusivamente como una estrategia de integración y de bienestar referido a un sistema benefactor. Se realizan entonces campeonatos y competencias en diversos contextos que se convierten rápidamente en repeticiones de la estructura deporte de rendimiento, aunque en menor escala. Prevalece la misma ideología que ha sostenido al deporte moderno desde sus orígenes. En virtud de este reduccionismo, se utiliza el deporte social comunitario como la simple y esporádica aplicación instrumental del deporte moderno, con todas sus características, a diferentes grupos de personas, aun cuando se argumente que por esta vía se desarrolla la calidad de vida.

Así mismo, específicamente en Colombia, como parte de propuestas incluidas en el deporte social comunitario, existen numerosos proyectos y programas en los que se evidencia una honda fractura entre los objetivos y los logros alcanzados (Hurtado, Restrepo, Martínez y Escobar, 2005). Además, se ha establecido que las actividades eminentemente deportivas relacionadas con campeonatos, promueven la agresividad, las luchas de poder y posiblemente las actitudes destructivas (Duarte Bajaña, Cáliz Romero, 2005). Sin embargo, y teniendo en cuenta la fundamentación desde las categorías bioéticas que se expone a continuación, el deporte social comunitario puede ser entendido como un nuevo paradigma, diferente y de más trascendencia que el deporte moderno en tanto que es un proceso de construcción colectiva y constante de tejido comunitario, que usa como herramienta el deporte y las transformaciones que hace de él para tener un acceso real a la promoción de la calidad de vida.

\section{El deporte social comunitario en función de la calidad de vida.}

Antes de abordar las categorías que pueden fundamentar el deporte social comunitario resulta necesario precisar sintéticamente el sentido de tres conceptos neurálgicos dentro de esta reflexión: Bioética, comunidad y calidad de vida.

Desde los inicios del siglo XXI se ha visto la necesidad de ampliar el discurso bioético para trascender los ámbitos clínicos y teniendo en cuenta campos relacionados con las ciencias sociales y medioambientales (Kottow, 2009). La Bioética puede entenderse como la dinamizadora de un nuevo modo de abordar las relaciones y comportamientos éticos respecto a la vida en el planeta (Zambrano, 2007). Dentro de este trabajo, se asume la vida como un proceso que trasciende lo exclusivamente fisiológico; se vive siempre y cuando existan procesos de relación en un contexto y la interacción con otros y con el ambiente le otorga sentidos a esa vida. La Bioética promueve el aprendizaje, la adquisición y el desarrollo consciente, individual, colectivo y paulatino de ciertas herramientas que permiten que aquellas interacciones sean cada vez más reflexivas y armónicas. Siendo así, la Bioética tiene profundas implicaciones teóricas pero además prácticas; es una praxis vital. La teoría y la práctica de la Bioética se ubican en torno a los problemas y circunstancias que atentan o interfieren con la vida, su calidad y sus sentidos. En este orden de ideas, las categorías que se mostrarán más adelante son estructurales para la Bioética.

En cuanto al concepto de comunidad, resulta valiosa la caracterización realizada por Ander Egg, para quien una comunidad es:

Una agrupación o conjunto de personas que habitan un espacio geográfico delimitado y delimitable, cuyos miembros tienen conciencia de pertenencia o de identificación con algún símbolo local y que interactúan entre sí más intensamente que en otro contexto, operando en redes de comunicación, intereses y apoyo mutuo, con el propósito de alcanzar determinados objetivos, satisfacer necesidades, resolver problemas o desempañar funciones sociales relevantes a nivel local (Egg, 1998: 27). 
Sin embargo, es necesario precisar que una comunidad es mucho más que un grupo de personas que vive en cierto lugar con unos intereses en común y cuyas relaciones son agradables y armoniosas. Esta visión de la comunidad no es real (Palacios, 1999). Son interesantes las concepciones recientes que de este término realizan las ciencias sociales según las cuales una comunidad no es un hecho según miradas externas, mas bien es un hecho que depende de dinámicas internas relacionadas con los vínculos, la cohesión, la consistencia interna, el reconocimiento de su misma existencia y de sus posibilidades, el sentido de pertenencia y sus propios intereses (Castro, 1998); es decir, una comunidad resulta siendo una estructura de poder. Siendo así, deben existir, o mejor, deben formularse, desarrollarse y construirse diferentes grados de libertad, según sea la comunidad, que permitan tantas posibilidades de vida como sean necesarias dentro del camino que busca la adaptación y la convivencia.

Teniendo en cuenta que el objeto central de este texto no es realizar un desarrollo amplio de los complejos significados que puede tener la calidad de vida, es preciso decir que aquí este concepto es entendido como lo plantea Boladeras: "la percepción del individuo de su situación en la vida y en el contexto de su cultura y el sistema de valores en que vive y en relación a sus objetivos, expectativas, estándares y preocupaciones" (2000: 34). Este planteamiento ha permitido entender mejor la calidad de vida y ha sido retomado y argumentado por varios autores como Maldonado (2000) y Escobar (2000).

Luego de aclarar estos tres conceptos vertebrales, expondremos sintéticamente, unos fundamentos que permiten sostener el deporte social comunitario para que responda a su objetivo primordial: la calidad de vida de los sujetos. Estas ideas se basan en las categorías ya mencionadas en la introducción de este texto, las cuales son constitutivas de la Bioética.

\section{La transdisciplinariedad: base para un deporte} social comunitario multisectorial

La Bioética, en su constitución teórica y en su práctica, se asume como un campo interdisciplinar; es decir, se asume como el abordaje de diversos dilemas en donde se ponen en juego la vida y la calidad de vida, que se realiza desde diferentes disciplinas, por cuanto la interdisciplinariedad se interesa por la dinámica generada por la acción simultánea de varios niveles de realidad (Martínez, 2003). La Bioética tiene un alcance que supera las barreras profesionales particulares (Engelgardt, 1995), dada la necesidad de rutas transdisciplinares. La transdisciplinariedad (Nicolescu, 1996) tiene en cuenta los vacíos existentes entre, a través y más allá de las disciplinas (Garrafa, 2005). La Bioética se inscribe entonces, en un espacio interdisciplinario y sobre todo transdisciplinario. Este proceso transdisciplinar permite formar agentes (Sen, 2010) con capacidad de analizar sus contextos de una forma amplia, lo que permite que diferentes disciplinas participen del desarrollo de su comunidad y posibilita la creación de miradas y posturas que faciliten la atención de sus necesidades.

En este proceso se busca que las propuestas de trabajo construidas en el marco del deporte social comunitario estén vinculadas a lo multisectorial (gremios, empresas, comercios, ONG's, universidades, grupos de base, juntas vecinales, municipio, organizaciones provinciales y nacionales, etc.) (Kisnerman, 2004). El deporte social comunitario se comprende como un proceso transdisciplinar que hace parte de una propuesta macro con características propias de la animación socio-cultural (Ander-Egg, 2001) y del trabajo social. Asume, además, que sólo a través de la educación, la participación (Ander-Egg, 2001; Bañez Tello, 2003; Chávez Carapia, 2003), un cierto grado de consenso y la percepción generalizada por parte de los individuos de estar personalmente comprometidos en la empresa, pueden lograrse avances y transformaciones comunitarias duraderas y satisfactorias (Gell-Mann, 1995).

El deporte social comunitario se asume entonces como un entramado educativo en el que la educación, como un proceso transdisciplinar, favorecería la formación de agentes (Sen, 2010) comprometidos con su realidad y con capacidad de analizar sus contextos de una forma amplia, lo que permite que diferentes disciplinas participen del desarrollo de su comunidad y en ocasiones posibilita la creación de nuevas disciplinas que puedan atender sus necesidades.

\section{Un deporte social comunitario incluyente a partir del pluralismo}

El pluralismo se encuentra como otra categoría de la Bioética a partir de la cual ya no se trata de enmarcar respuestas dentro de una tradición particular, sino que las formula de manera abierta para individuos racionales. La Bioética ayuda a clarificar diversas visiones de la realidad y los valores (Engelhardt, 1995). Desde aquí, es factible reconocer las posiciones en conflicto (varias posiciones frente a un problema) y generar 
procesos que permitan que los actores se interesen por las diferencias y similitudes entre ellos y los otros.

Desde el pluralismo, el deporte social comunitario tiene en cuenta los sistemas morales que se tejen dentro de la comunidad y promueve escenarios para que la reflexión ética pueda darse; reconoce que en una comunidad existen posiciones en conflicto y se interesa por las diferencias y similitudes que generan esos conflictos para comprender $y$, en consecuencia, producir acercamientos desde la reflexión (Riso, 2006).

En este sentido, el deporte social comunitario respeta la diversidad (Gell-Mann, 1995), por medio de la cual resulta posible comprender y asumir que los participantes no son iguales y aún así es posible aprender de ellos promoviendo el desarrollo de una actitud, una forma de ser que le permita a los sujetos comprender lo ya mencionado: sus diferencias y similitudes y, como resultado, producir acercamientos empáticos.

El deporte social comunitario fundamentado en las categorías bioéticas puede ser entendido como un escenario de inclusión (Lleixà, 2004), en el que los diferentes, más que integrarse, deben ser incluidos, entendiendo que la integración requiere de una adaptación continua de los participantes a unas creencias, normas, valores o principios hegemónicos (Rubio-Carracedo, 2003), mientras que la inclusión evidencia el pluralismo dentro de un proyecto social común que se transforma según sean las necesidades de la población (Bertrán, Echeita Sarrionandia, Sandoval Mena, López, Durán, Giné Giné, 2002, Abery, 2006).

La articulación reflexión-diálogo-deliberación dentro del deporte social comunitario: posibilidades para la convivencia

La Bioética, mediante categorías como la reflexión, la deliberación y el diálogo y usando herramientas como la biopolítica (Maldonado, 1998, Kottow, 2007), busca el respeto a la vida en todas sus formas, pues comprende que toda forma de vida lleva implícita un proceso de aprendizaje, un camino en pos de la adaptación y la acomodación. La reflexión, como categoría Bioética, permite que el deporte social comunitario se constituya en un medio para que los sujetos hagan conciencia ampliada (Damasio, 2001) y fenomenológica (Chalmers, 1999) de su calidad de vida. La reflexión no es un proceso puramente racional, cerebral o fisiológico; en el deporte social comunitario esta categoría está atravesada por la emoción, la acción y el dialogo. Aquí el lenguaje promueve la capacidad de coordinarse con el otro para generar acciones. En términos de Maturana (1997) el lenguajear consiste en operar en coordinaciones de coordinaciones conductuales consensuales recurrentes, es decir, vivir en el lenguaje o vivir el lenguaje, el cual está permanentemente articulado a la emoción. Es este emocionar el que le otorga tintes relacionados con la convivencia a las conversaciones de los participantes del deporte social comunitario. En este orden de ideas, los diálogos interculturales son fundamentales ante el dilema de la pluralidad de mundos morales que pueden convivir en un mismo grupo que se esté tejiendo como comunidad (Zambrano, 2007)

Ahora bien, esta relación entre reflexión, dialogo y emoción, no significa, en ningún caso, ubicar el deporte social comunitario en un relativismo moral. Las reflexiones, conversaciones y acciones que allí ocurren están signadas, como se expuso arriba, por un pluralismo ético. Según Riso, existen cuatro diferencias importantes entre estos dos conceptos:

El pluralismo requiere de la justificación de los sistemas morales para que la reflexión ética pueda darse; el relativismo asume que todos son igualmente válidos. En segundo lugar, el pluralismo reconoce las posiciones en conflicto (varias posiciones frente a un problema); en el relativismo no se plantean problemas. En tercer lugar, la actitud pluralista se interesa por las diferencias y similitudes para comprender y en consecuencia producir acercamientos; el relativismo se fundamenta en el "todo vale" y por tanto hace una apología al "respeto" pero desde la indiferencia, lo cual no deja de ser una contradicción si concebimos el respeto como la escucha activa (interesada) del otro. Finalmente en el pluralismo se ponen en riesgo las propias posiciones en tanto se acepta cualquier transformación posible de las partes; el relativismo no trasciende de la "tolerancia" ya que no hay nada que cambiar (Riso, 2006: 30).

La reflexión y el dialogo, así entendidos, permiten la construcción de un deporte social comunitario que promueva en los participantes la capacidad de coordinarse con el otro para generar acciones concientes. Este proceso implica tener en cuenta la deliberación, que permite que los sujetos propongan y construyan herramientas para la generación de procesos comunicativos que faciliten la negociación y el diálogo, entendiendo este tránsito comunicativo como síntesis dialéctica de ideas y criterios que produce puestas en común y permite la producción de elementos de convivencia. El diálogo, como categoría bioética, es entonces un motor fundamental dentro del desarrollo de la 
calidad de vida al permitir que los participantes del deporte social comunitario expresen - lo cual requiere de otro u otros empáticos con esa expresión - sus percepciones frente a su situación en la vida, expresen su sentir frente al contexto que están construyendo, expresen sus acuerdos o desacuerdos respecto al sistema de valores en que viven y expresen sus ideas y emociones en relación a sus objetivos, expectativas, y preocupaciones. Por este camino el diálogo puede entenderse como una herramienta para la creación continua de comunidad, a través de procesos deliberativos que abran senderos hacia la convivencia superando el aislamiento y la sumisión (López Forero, 1999).

\section{El respeto por diferentes grados de libertad en el deporte social comunitario y el desarrollo de capacidades}

Para abordar esta categoría, es necesario decir que las personas que participan y aprenden del deporte social comunitario, viven en el mundo de hoy, un mundo de incertidumbres, sin marcos claros de referencia; un mundo en el que la idea de crisis expresa la normalidad de cualquier ámbito social (Barbero González, 2005). En este marco, la Bioética busca y plantea diferentes grados de libertad. Esta categoría bioética implica diferentes maneras de entender el mundo y diferentes formas de acceder y desarrollar las capacidades, entendiéndose capacidad (Sen, 2010) como las combinaciones alternativas de funcionamientos vitales por medio de los cuales una persona puede elegir la vida que tendrá. En este sentido, la capacidad de una persona corresponde a la libertad que tiene de llevar una determinada clase de vida (Nussbaum, Sen, 2002).

Con el ánimo de aclarar este punto, y siguiendo los planteamientos de Amartya Sen (2010), las libertades civiles y políticas son constitutivas del concepto de libertad humana, su ausencia implica por lo tanto, un handicap. En este orden de ideas, la calidad de vida no se puede reducir a la evaluación de las condiciones económicas; se refiere a las posibilidades que tienen los seres humanos para trabajar, mejorar y desarrollar sus capacidades con base en sus necesidades, en un marco de libertad, es decir, de oportunidades y de participación (Ander-Egg, 2001; Bañez Tello, 2003; Chávez Carapia, 2003). Los sujetos individuales, aunque organizados social y políticamente, tienen una gran responsabilidad en la construcción de esas libertades, lo cual generará más capacidades en los sujetos, esto es, más oportunidades para desarrollar su calidad de vida.
El deporte social comunitario debe ubicarse en un territorio axiológico diferente al deporte moderno. El fin último deja de ser ganar en el sentido de alcanzar más puntos que los contrincantes. Esta finalidad puede pasar a ser una herramienta en aquellos casos (realmente esporádicos) en donde las necesidades de las poblaciones requieran dentro de sus procesos de desarrollo, actividades que impliquen rivalidad. Desde este argumento, la bioética y sus categorías permiten aseverar que la ganancia real de los procesos generados desde el deporte social comunitario se ubica en las capacidades y libertades que la comunidad adquiera y desarrolle así como en las percepciones que sus miembros tengan de su propia vida y de su contexto. Entonces, la búsqueda del guerrero, la búsqueda de técnicas, tácticas y estrategias deportivas, la búsqueda del éxito, del triunfo, de la gloria, de ser el número uno, de estar en un nivel superior a todos los demás, la búsqueda de escalar posiciones per se y la búsqueda de medallas y trofeos se desvanece porque pierde su sentido vital. Lo que está en juego en el deporte social comunitario es la construcción de libertades, de capacidades, de calidad de vida.

\section{Un deporte social comunitario colectivo y contextual}

Finalmente, existen dos categorías bioéticas que permiten fundamentar el deporte social comunitario: el trabajo colectivo y la contextualización. Desde la Bioética se entiende la colectividad como un escenario desde donde se abordan los dilemas y las propuestas, se otorga gran importancia a una conciencia supraindividual y no solamente a una conciencia individual (Maldonado, 2000). La Bioética carece de normas universalmente válidas, pero hace uso de principios de aceptación general, aunque no absolutos (Kottow, 2005). Esta idea permite empezar a comprender la categoría de la contextualidad. La Bioética tiene en cuenta las características, las necesidades y las problemáticas propias de la población con la que se va a interactuar; cada proceso es único y responde a unas características propias, diferentes de otros grupos poblacionales.

Los objetivos del deporte social comunitario deben plantearse en colectivo; las comunidades ayudan a diseñarlos, lo que facilita su pertinencia y su futuro cumplimiento. Estos objetivos, además, deben descentrarse de la búsqueda de esparcimiento, recreación y desarrollo físico. El deporte social comunitario utiliza como enlaces estas características para favorecer la 
calidad de vida dentro de las comunidades. Es decir, estas características además de ser un fin en sí mismas son medios: son fines y herramientas de deporte social comunitario.

Fundamentalmente, el deporte social comunitario debe superar dentro de sus objetivos el sistema axiológico propio del deporte moderno; en cambio, se ubica dentro de un sistema de valores propio de la relación poder-ética que subyace al contexto en donde va a participar. Esto lo convierte en un proceso generado en torno al deporte social comunitario que sea replicado en otro contexto sin tener en cuenta las características, necesidades y problemáticas propias de la población con la que se va a interactuar; puede resultar inútil o contraproducente, es decir, cada proceso es único y responde a unas características propias, diferentes de otros grupos poblacionales. El deporte social comunitario no tiene un programa prefijado diseñado desde el desconocimiento de la existencia de diversas realidades, se transforma a partir de las necesidades de cada contexto.

\section{Tres conceptos de la bioética como aporte al de- porte social comunitario}

Concluimos que la Bioética se configura gracias a las categorías expuestas anteriormente, entre otros aspectos. Esa estructuración le permite construir algunos conceptos que también pueden ayudar a estructurar el deporte social comunitario. Se plantean aquí tres de ellos: en primer término, la idea de extraños morales (Engelhardt, 1995). Por esta vía se hace posible reflexionar acerca de justificar un marco moral por medio del cual los individuos pertenecientes a comunidades morales diferentes puedan considerarse vinculados por una estructura moral común y puedan apelar a una Bioética también común. El segundo concepto que le aporta la Bioética al deporte social comunitario es el principio de igual consideración de intereses (Singer, 2010), por medio del cual en todas las actividades que se lleven a cabo se puede tener la posibilidad de reflexionar acerca de los intereses de la población participante en esas actividades y de cómo estos intereses pueden ser logrados de manera equitativa. El tercer concepto fundamental es el de comunidad de orden superior (Engelhardt, 1995) con el que se supera el concepto de comunidades cerradas y se accede a la necesidad de reunir a varias comunidades, en torno a objetivos y tareas comunes.
Las categorías bioéticas y conceptos construidos por la Bioética permiten convertir al deporte social comunitario en un escenario de inclusión en donde los diferentes, más que integrarse, deben ser incluidos. El deporte social comunitario, alimentado desde la Bioética, empieza a ser comprendido como un territorio axiológico diferente al deporte moderno. El fin último deja de ser ganar a toda costa y pasa a ser una herramienta al servicio de la calidad de vida en un marco comunitario.

\section{A manera de síntesis y apertura}

Evidentemente, siguiendo la fundamentación anterior, el concepto de comunidad al que debe responder el deporte social comunitario sufre un vuelco fundamental desde una mirada Bioética; las comunidades deben ser entendidas como organizaciones abiertas, nunca cerradas, pues se corre el riesgo de perderse y desaparecer en el mundo de incertidumbres contemporáneo. Es en este sentido que cobra gran importancia el concepto de comunidad de orden superior planteado por Engelhardt (1995).

Un concepto que ilustra bellamente el marco en el cual se mueven las comunidades a las que se dirige el deporte social comunitario es el de culturas híbridas (García Canclini, 1990). Vale la pena recordar que la cultura popular puede ser entendida como un proceso de invención dirigido al presente que se realiza mediante hibridaciones complejas que penetran en todas las clases, etnias y fronteras nacionales. Esta hibridación genera permanentemente realidades negociadas en contextos determinados por tradiciones, capitalismo y modernidad (Escobar, 1996).

Detrás de este planteamiento existe un fuerte contenido bioético, pues la hibridación cultural evidencia una salida a la polarización entre lo universal y lo local, en donde los extraños morales hacen acuerdos de coexistencia en un marco plural configurando nuevas realidades. El deporte social comunitario puede ser entendido como una herramienta que promueve procesos de organización social con el ánimo de que, de manera reflexiva y consciente, una parte de la población aprenda a controlar sus problemas y lograr mejores y mayores servicios (Lindeman, 1921), para lo cual deben existir negociaciones y acuerdos entre extraños morales.

Con este marco de referencia, es posible establecer relaciones entre comunidad y deporte, diferenciando esta articulación del deporte moderno - estructura 
axiológica con una base europea de los siglos XVIII y XIX - y denominando a ese empalme como deporte social comunitario, concepto entendido a la luz de las categorías bioéticas, como un ámbito transdisciplinario y plural que sirve como herramienta para que las comunidades generen procesos de reflexión, deliberación y construcción de redes de comunicación. El deporte social comunitario desde esta fundamentación puede convertirse en un proceso de aprendizaje que permite desarrollar la percepción que los individuos participantes

\section{Referencias}

Abery, B. (2006) Ways to Enhance Social Inclusion. Impact. Volumen 19(2) 12.

Ander-Egg, E. (1998) Metodología y práctica del desarrollo de la comunidad. Buenos Aires: Lumen-Humanitas.

(2001) Metodología y práctica de la animación sociocultural. Madrid: Instituto de Ciencias Sociales Aplicadas.

Bañez Tello, T. (2003) Ciudadanía y Participación. En Benruz Beneites, M. y Susín B., (2003): Ciudadanía: dinámicas de pertenencia y exclusión. Universidad de la Rioja, Servicio de Publicaciones. 97-112.

Barbero González. J. I. (2005) La escolarización del cuerpo: Reflexiones en torno a la levedad de los valores del capital «cuerpo» en educación física. Revista iberoamericana de educación. 39, 25-51.

Boladeras, M. (2000) Calidad de vida y principios bioéticos, En Boladeras, M. et. al. Bioética y calidad de vida, Bogotá: El Bosque, colección Bios y Ethos, volumen 15, 21-54.

Bertrán, E. M., Echeita Sarrionandia, G., Sandoval Mena, M., López, M. L., Durán, D., Giné Giné, C. (2002) Index for Inclusión: Una guía para la evaluación y mejora de la educación inclusiva. Contextos educativos: Revista de educación. 5. p. 227-238.

Brohm, J. (1982) Sociología política del deporte, México: Fondo de cultura económica.

Castro, M. C., et. al. (1998) Psicología, Educación y Comunidad, Bogotá: Almudena Editores.

Chalmers, D. J. (1999) La mente consciente. Barcelona: Gedisa.

Constitución Política De Colombia. (1995) Ley 181, artículo 16

Chávez Carapia, J. (2003) La participación social: retos y perspectivas, México: UNAM, Escuela Nacional de Trabajo Social.

Damasio, A. (2001) La sensación de lo que ocurre, cuerpo y emoción en la construcción de la consciencia, Barcelona: Debate.

Duarte Bajaña, R., Cáliz Romero N. (2005) Actividad física, recreación y deporte en la calidad de vida de un grupo de mujeres de la cárcel El Buen Pastor, Bogotá: Revista U.D.C.A. 8(1) 13-24. tienen de su situación en la vida y en el contexto de su cultura, así como del sistema de valores en que viven en relación con sus objetivos, expectativas, estándares y preocupaciones.

En próximos trabajos se abordarán las tareas fundamentales que le competen al deporte social comunitario teniendo en cuenta la fundamentación que aquí se ha bosquejado.

Engelhardt, T. (1995) Los fundamentos de la bioética Barcelona: Paidós.

Escobar, A. (1996) La invención del tercer mundo. Bogotá: Norma.

Escobar, J. (2000) Comprensión sistémica de la salud y calidad de vida. Bioética y calidad de vida. Bogotá: Ediciones en Bosque, colección Bios y Ethos. 15, 55-75.

Foucault, M., (1994) Genealogía del racismo. Madrid: La Piqueta.

García Canclini, N., (1990) Culturas híbridas: estrategias para entrar y salir de la modernidad. México: Grijalbo.

Garrafa, V. (2005) Multi-inter-transdisciplinariedad, complejidady totalidad concreta en bioética. En Garrafa, V., Kottow, M., Saada, A. Estatuto epistemológico de la Bioética, México: UNAM, Redbioética, UNESCO, 67-86.

Gell-Mann, M. (1995) El quark y el jaguar, aventuras de lo simple a lo complejo. Barcelona: Tusquets.

González, T. (2002) La política deportiva en España durante la república y el franquismo. Sport y autoritarismos: la utilización del deporte por el comunismo y el fascismo, Madrid: Alianza. p. 169-202.

Hurtado, D., Restrepo, A., Martínez, M., Escobar, F. (2005) Experiencias deportivas y/o recreativas que en medio de contextos de violencia aportan a la construcción de tejido social y a la convivencia en Medellín. Medellín: INDER, Instituto de Estudios Políticos de la Universidad de Antioquia.

Kisnerman, N. (septiembre, 2004) Desafíos del trabajo comunitario. Ponencia presentada en el "IV encuentro internacional prospectiva del desarrollo comunitario sostenible por áreas de intervención". Bogotá: Universidad Colegio Mayor de Cundinamarca.

Kottow, M. (2005) Introducción a La Bioética. Chile: Mediterráneo.

(2007) Ética de protección, una propuesta de protección Bioética. Bogotá: Universidad Nacional de Colombia, Bosque

(2009) Bioética ecológica. Bogotá: Universidad El

indeman, E. (1921) The Community: An Introduction to the Study of Community. New York: Association Press. Lleixà, T. (2004) Actividad física, deporte y ciudadanía 
intercultural. En: Lleixà, Teresa, Soler, Susana. Actividad física y deporte en sociedades multiculturales. ¿integración o segregación?, Barcelona: Horsori, 1326.

López Forero, L. (1999) La comunicación en la comunidad. En: González, L., Vargas, G., Betancourt, D., Houhton, T. Barreto, M., et.al. Organización y promoción de la comunidad, Bogotá: El búho, 127-154.

Maldonado, C. (1998) Biopolítica de la guerra, Colombia: Siglo del hombre editores. (2000) Fundamentos para la comprensión del problema de la calidad de vida, En: Bioética, la calidad de vida en el siglo XXI, Bogotá: El Bosque, colección Bios y Ethos. 1.

Mandell, R. (1986) Historia Cultural del deporte. Barcelona: Bellaterra.

Martínez, M. Transdisciplinariedad. (2003) Un enfoque para la complejidad del mundo actual. Revista Concienciactiva21, Caracas. 1, 107-146.

Maturana, H. (1997) Emociones y lenguaje en educación y política. Santiago de Chile: Dolmen.

Nicolescu, B. (1996) La transdisciplinariedad-manifiesto, Monaco: Editions du Rocher.

Nussbaum, M, Sen, A. (2002) La calidad de vida. México: Fondo de cultura económica.

Palacios, N. (1999) Organización y trabajo comunitario. En González, J. Organización y promoción de la comunidad, Bogotá: El búho, 209-222.
Riso, W. (2006) ¿Es posible una bioética anclada en el egocentrismo?: hacia una mente pluralista, interdisciplinaria y abierta al cambio. Tesis, Maestría en Bioética. Bogotá: Universidad el Bosque.

Rubio-Carracedo, J. (2003) Pluralismo, multiculturalismo y ciudadanía. En: Badillo O'Farrell, P. Pluralismo, tolerancia, multiculturalismo: reflexiones para un mundo plural. España: Akal, 173-194

Ruiz Aguilera, R. (1991) El deporte de hoy, realidades y perspectivas. La Habana: Editorial Científico Técnica.

Sen, A. (2010) Libertad y desarrollo. México: Fondo de cultura económica.

Singer, P. (2010) Ética práctica. Cambridge: University Press.

Teja, Á. (2002) Deporte y relaciones internacionales durante el fascismo en Italia. En: González, T. Sport y autoritarismos: la utilización del deporte por el comunismo y el fascismo, Madrid: Alianza, 241-280.

Velázquez Buendía, R. (2001) El deporte moderno. Consideraciones acerca de su génesis y de la evolución de su significado y funciones sociales. Revista Educación Física y deportes [online]. Buenos Aires. 36. Recuperado el 7 de julio de 2011 en: http://www. efdeportes.com/efd36/deporte.htm

Zambrano Rodriguez, C. V. (2007) Bioética en la transición cultural. En: Novoa, E., Lesport, I., De Zubiría, S., Ovalle, C., Zamabrano, C. Bioética, desarrollo humano y educación. Bogotá: Universidad El Bosque, 175-215. 